\title{
EXTENSÃO UNIVERSITÁRIA: FORMAS DE UTILIZAÇÃO E OPÇÕES PARA DESCARTE DE ÓLEOS COMESTÍVEIS
}

\author{
Maria Bruna de Souza Mulinari \\ https://orcid.org/0000-0001-6068-9485 \\ Eliane FerReira de Souza \\ https://orcid.org/0000-0002-9721-4464 \\ Mikaelly Nayara Santos \\ https://orcid.org/0000-0001-8923-7124
}

\section{Cinthia Aparecida de Andrade Silva \\ https://orcid.org/0000-0001-9070-1182}

\section{Antonio Rogério FioruCCi ${ }^{1 *}$ \\ https://orcid.org/0000-0001-9441-1561}

Resumo: O Brasil possui grande diversidade de plantas oleaginosas das quais é possível extrair óleos comestíveis. Esses óleos vegetais são importantes fontes energéticas, sendo constituídos principalmente de glicerídeos, podendo conter pequenas quantidades de fosfolipídios e ácidos graxos livres. O processo de aquecimento durante o preparo dos alimentos provoca degradação oxidativa e modificações físico-químicas nos óleos vegetais, algumas das quais são perceptíveis sensorialmente como o escurecimento, o aumento da viscosidade, a formação de espuma e a fumaça. Durante esse tipo de deterioração, são formados peróxidos e hidroperóxidos, que por sua vez, originam compostos voláteis, como aldeídos, cetonas e ácidos carboxílicos. Essas substâncias passam a fazer parte dos alimentos preparados podendo ser tóxicas e prejudiciais à saúde dos consumidores, consideradas possíveis causas do desenvolvimento de doenças e envelhecimento precoce. O objetivo deste trabalho é orientar a população sobre as formas de consumo de óleos vegetais comestíveis por meio de palestras informativas e aplicação de questionário sócio econômico enfatizando também os efeitos que o descarte incorreto dos mesmos pode causar ao meio ambiente. As palestras informativas aos alunos evidenciaram

1 Universidade Estadual de Mato Grosso do Sul, Centro de Estudos em Recursos Naturais (CERNA).

*Autor correspondente . E-mail: arfioruci@yahoo.com.br 
uma necessidade de orientação sobre uma correta forma de utilização dos óleos comestíveis no preparo cotidiano de alimentos. Com a análise das respostas ao questionário evidenciou-se que a maioria dos entrevistados nos grupos analisados (57 a 90\%) aproveitam o óleo residual utilizado no preparo de alimentos para fabricação de sabão; esta é uma solução para o descarte inadequado evitando danos ao meio ambiente.

Palavras-Chave: Aquecimento, Alimentos, Estabilidade oxidativa.

\title{
UNIVERSITY EXTENSION: FORMS OF USE AND OPTIONS FOR DISPOSAL OF EDIBLE OILS
}

\begin{abstract}
Brazil has a great diversity of oil producing plants from which it is possible to extract edible oils. These vegetable oils are important energy sources, consisting mainly of glycerides, and may contain small amounts of phospholipids and free fatty acids. The heating process during food preparation causes oxidative degradation and physico-chemical modifications in vegetable oils, some of which are sensorially perceptible as darkening, viscosity increase, foaming and smoke. During this type of deterioration, peroxides and hydroperoxides are formed which, in turn, give rise to volatile compounds, such as aldehydes, ketones and carboxylic acids. These substances become part of prepared foods that can be toxic and harmful to the health of consumers, considered possible causes of disease development and premature aging. The objective of this work is to guide the population about the consumption of edible vegetable oils through informative lectures and the application of a socioeconomic questionnaire emphasizing also the effects that the incorrect disposal of them can cause to the environment. The informative lectures to the students evidenced a need for guidance on a correct way of using edible oils in the daily food preparation. With the analysis of the questionnaire responses, it was evidenced that the majority of the interviewees in the analyzed groups (57 to 90\%) took advantage of the residual oil used in the preparation of food for soap production; this is a solution for the proper disposal, avoiding damage to the environment.
\end{abstract}

Keywords: Heating, Food, Oxidative stability.

\section{EXTENSIÓN UNIVERSITARIA: FORMAS DE UTILIZACIÓN Y OPCIONES PARA DESCARTE DE ACEITES COMESTIBLES}

Resumen: Brasil poseé gran diversidad de plantas oleaginosas de las cúales es posible extraer aceites comestibles. Estos aceites vegetales son importantes fuentes energéticas, constituidos principalmente de gliceridos, pudiendo contener pequeñas cantidades de fosfolípidos y ácidos grasos libres. El proceso de calentamiento durante la preparación de los alimentos provoca degradación oxidativa y modificaciones físico-químicas en los aceites vegetales, algunas de las cuales son perceptibles sensorialmente como el oscurecimiento, el aumento de la viscosidad, la formación de espuma y el humo. Durante este tipo de deterioro, se forman peróxidos e hidroperóxidos, que a su vez, originan compuestos volátiles, como aldehídos, cetonas y ácidos carboxílicos. Estas sustancias pasan a formar parte de los alimentos preparados, pudiendo ser tóxicos y perjudiciales para la salud de los consumidores, considerados posibles causas del desarrollo de enfermedades y envejecimiento precoz. El objetivo de este trabajo es orientar a la población sobre las formas de consumo de aceites vegetales comestibles por medio de charlas informativas y aplicación de cuestionario socio económico enfatizando también los 
efectos que el descarte incorrecto de los mismos puede causar al medio ambiente. Las charlas informativas a los alumnos evidenciaron una necesidad de orientación sobre una correcta forma de utilización de los aceites comestibles en la preparación cotidiana de alimentos. Con el análisis de las respuestas al cuestionario se evidenció que la mayoría de los entrevistados en los grupos analizados (57 a 90\%) aprovechan el aceite residual utilizado en la preparación de alimentos para la fabricación de jabón; esta es una solución para el descarte inadecuado evitando daños al medio ambiente.

Palabras clave: Calefacción, Alimentos, Estabilidad oxidativa

Submetido em: 02/12/2018

Aceito em: 22/12/2018

\section{INTRODUÇÃO}

Uma alimentação balanceada e saudável, além da prática de exercícios físicos regulares são hábitos a serem adotados para garantir uma boa saúde. Segundo o Ministério da Saúde (2013), os brasileiros, nos últimos anos, passaram por mudanças sociais que modificaram seus padrões de saúde e consumo alimentar. Essas mudanças acarretaram uma diminuição da pobreza e da exclusão social e, consequentemente, da fome e desnutrição. Contudo, observa-se o aumento acelerado do excesso de peso em todos os níveis sociais, assinalando um novo cenário de problemas relacionados à alimentação e nutrição, principalmente em decorrência do consumo exagerado de alimentos ricos em açucares e lipídios.

Os lipídios são moléculas orgânicas que fazem parte da composição de inúmeros alimentos, formadas a partir de ácidos graxos e álcool, e desempenham importantes funções no organismo dos seres vivos (FOOD INGREDIENTS BRASIL, 2016), dentre elas o transporte de vitaminas sendo considerados importantes fontes energéticas:

Os lipídios são os principais depósitos de energia. Constituem o combustível celular ideal, pois cada molécula carreia grandes quantidades de energia por unidade de peso podendo ser encontrados livres nas células como reserva energética. Os ácidos graxos são as biomoléculas mais calóricas, apesar dos carboidratos serem bem mais eficazes na produção de energia (FOOD INGREDIENTS BRASIL, p. 56, 2016).

O Brasil possui grande diversidade de plantas oleaginosas das quais é possível extrair óleos. Esses óleos vegetais são importantes fontes energéticas, 
sendo constituídos principalmente de glicerídeos, podendo conter pequenas quantidades de fosfolipídios e ácidos graxos livres (ANVISA, 2017).

A cada ano observa-se um aumento no consumo e aceitação de alimentos processados por frituras. O processo consiste em inserir o alimento em oléo ou gordura a uma temperatura alta. No entanto, esta técnica culinária, que parece tão simples, é um processo extraordinariamente complexo, em que se encontram envolvidos vários fatores (CORSINI \& JORGE, 2006).

O processo de fritura provoca modificações físico-químicas nos óleos vegetais, algumas das quais são visíveis como, o escurecimento, aumento da viscosidade e formação de espuma (REDA e CARNEIRO, 2007). A estabilidade oxidativa é um importante parâmetro utilizado para avaliação da qualidade de um óleo comestível (TAN et. al. 2002) e a diferença desta propriedade para os diferentes tipos de óleos está relacionada principalmente com a presença de ácidos graxos poli-insaturados e com a quantidade de antioxidantes naturais como os tocoferóis.

O método Rancimat é um teste de oxidação acelerada que permite caracterizar o estado de conservação de óleos e gorduras com facilidade e reprodutibilidade dos resultados (FARHOOSH e MOOSAVI, 2007) e consiste em expor a amostra a um fluxo de ar e temperatura estabelecidos. Durante o decorrer da análise, esses fatores favorecem a deterioração do material analisado formando peróxidos e hidroperóxidos, que por sua vez, originam compostos voláteis, como aldeídos, cetonas e ácidos carboxílicos de baixa massa molecular. Algumas dessas substâncias podem ser tóxicas e prejudiciais à saúde do consumidor, levando ao desenvolvimento de doenças cardiovasculares, artrite, envelhecimento precoce (MENDONÇA, et. al. 2008 e FIRESTONE, STIER, BLUMENTHAL, 1991), ação mutagênica e carcinogênica (FARHOOSH, EINAFSHAR, SHARAYEI, 2009).

Em virtude disto, é importante que ao adquirir um óleo comestível tenha-se conhecimento das mudanças e alterações que poderão influenciar sua qualidade, devido a interferência de fatores como a temperatura e exposição a luz, por exemplo, a fim de promover não só a proteção da saúde pública, mas o aumento da vida de prateleira do produto.

Vale ressaltar ainda que uma parte do óleo utilizado como meio de transferência de calor durante o preparo dos alimentos é absorvida pelos mesmos, tornando-se um ingrediente do produto de consumo humano. Nesse processo de aquecimento, ao longo do tempo, ocorre quebra de ligações de ésteres de 
ácidos graxos insaturados que fazem parte da composição dos óleos vegetais, ocorrendo a formação de ácidos graxos saturados um dos responsáveis pela diminuição da qualidade nutricional dos óleos comestíveis (OSAWA, GONÇALVES \& MENDES, 2010). É imprescindível que ao adquirir um óleo comestível, o consumidor saiba dos benefícios nutricionais e das suas limitações, visando a sua correta utilização na preparação de alimentos e os efeitos que o resíduo destes podem causar ao meio ambiente quando descartados de maneira incorreta.

O objetivo do presente trabalho foi orientar por meio de palestras informativas a população sobre as formas de consumo de óleos vegetais comestíveis bem como os efeitos que seu descarte incorreto pode causar ao meio ambiente, seguida da aplicação de questionário sócio econômico para identificar o perfil do consumidor de óleos comestíveis em Dourados-MS.

\section{METODOLOGIA}

Foi realizada uma pesquisa bibliográfica sobre o tema desde a composição dos óleos vegetais comestíveis até os efeitos causados a saúde e ao meio ambiente em decorrência de uma excessiva utilização destes, bem como as consequências do descarte incorreto do resíduo gerado ao meio ambiente.

Como dados de apoio para a elaboração das palestras informativas sobre as formas de consumo dos óleos vegetais e as implicações ambientais do seu descarte, utilizaram-se os resultados de estabilidade oxidativa para amostras de óleo obtidas no comércio local e analisadas no Analisador de Estabilidade Oxidativa, modelo PROFESSIONAL BIODIESEL RANCIMAT 893 (METRHOM, Suíça) do Centro de Estudos em Recursos Naturas (CERNA-UEMS). Para tais análises, empregou-se o Método de Rancimat para amostras comerciais de óleo de girassol, soja, milho, canola e oliva, seguindo as condições de análise (temperatura de $110^{\circ} \mathrm{C}$ e fluxo de ar de $10 \mathrm{~L} / \mathrm{h}$ ) estabelecidas pela norma AOCS Cd 12b-92. Os testes de oxidação acelerada (Rancimat), em duplicata, para as amostras forneceram dados de estabilidade oxidativa na forma de índice de estabilidade oxidativa (OSI) divulgados nas palestras por sua média ( \pm desvio padrão).

Elaborou-se um questionário estruturado com questões objetivas fechadas e abertas, aplicado de forma imparcial pelo entrevistador em escolas públicas e privada (para alunos maiores de 18 anos ou seus responsáveis) e no campus de Dourados da Universidade Estadual de Mato Grosso do Sul (para alunos de 
graduação). As questões fechadas ofereceram múltipla escolha ou dicotomia ( $\operatorname{sim} /$ não), havendo questões de resposta única e outras que permitem a seleção de múltiplas opções. As questões abertas foram de forma direta e solicitavam respostas curtas, apenas para nomeação das atividades, não havendo viés de discussão ou emissão de opiniões. Foram realizadas avaliações quantitativas de todas as repostas do questionário originando frequências e correlações calculadas, sendo estas representadas em forma de gráficos.

Para Mello e Bianchi (2015), o questionário é uma ferramenta para colher dados dentro do estudo. E como tal, sua função não é diminuída, ao contrário, passa a ser o motor que propulsiona o andamento do estudo. Algumas pesquisas só podem ser realizadas através de questionários como meio de coleta de dados.

\section{RESULTADOS E DISCUSSÃO}

3.1 Estabilidade oxidativa de óleos vegetais

Os resultados das análises de estabilidade oxidativa pelo método de Rancimat de óleos vegetais comestíveis de girassol, soja, milho, canola e oliva, adquiridos no comércio local, estão apresentados na Tabela 1.

TABela 1. VAlores de OSI obtidos Pelo teste de estabilidade oxidativa ACELERADA (RANCIMAT) DE AMOSTRAS DE ÓlEOS VEGETAIS COMERCIAIS.

\begin{tabular}{c|c|c|}
\hline ÓLEO VEGETAL & MARCA & OSI (H) \\
\hline Canola & A & $17,29 \pm 0,09$ \\
\hline Girassol & B & $10,03 \pm 0,02$ \\
\hline Milho & A & $5,61 \pm 0,01$ \\
\hline & B & $6,89 \pm 0,02$ \\
\hline Soja & A & $12,34 \pm 0,44$ \\
& B & $10,75 \pm 0,05$ \\
Azeite extra virgem & C & $12,90 \pm 0,17$ \\
& D & $6,41 \pm 0,08$ \\
\hline
\end{tabular}

Pode-se observar pelos resultados médios das análises laboratoriais para duas amostras do mesmo óleo vegetal que o óleo de canola é o óleo vegetal com maior estabilidade oxidativa (13,66 h), seguido do óleo de milho (11,55 h) e na sequência os óleos de soja $(9,66 \mathrm{~h})$, azeite $(6,52 \mathrm{~h})$ e o girassol $(6,25$ 
h). O OSI é um importante indicador da capacidade de degradação dos óleos. Quanto maior a quantidade de compostos insaturados no óleo, menor será o valor do OSI e maior a sua suscetibilidade a oxidação térmica (OSAWA, GONÇALVES \& MENDES, 2010; BODOIRA et al., 2017). Os resultados apresentados de cada óleo comprovam que a composição química do óleo vegetal influencia na sua estabilidade oxidativa.

A estabilidade térmica de um óleo depende da quantidade de saturações e instaurações que compõe a sua formulação (SANTOS, et.al. 2013), de maneira que os ácidos graxos insaturados são mais sensíveis à oxidação do que os saturados (SANTOS, 2008). Ácidos graxos livres e outras substâncias, tais como carotenóides, esteróis, fosfolipídios, substâncias fenólicas e tocoferol, foram apontados também como responsáveis pela estabilidade térmica (SANTOS, et.al. 2013).

Segundo a ANVISA, a utilização de óleos vegetais que possuem elevado índice de ácidos graxos com 2 ou 3 insaturações acabam por resultar em menor estabilidade oxidativa devido a presença de carbonos alílicos ou bís-alílicos. No caso dos óleos analisados (Tabela 1), o óleo de girassol, que possui entre 55 a 75\% de ácido linoleico (ômega-6 com 18 carbonos e duas insaturações em sua cadeia), foi o que apresentou menor estabilidade à oxidação. Para frituras, que é um dos processos mais empregados na área de alimentos, o óleo deve possuir um alto grau de estabilidade oxidativa em temperaturas elevadas. Por meio dos resultados apresentados anteriormente pode-se concluir que os óleos de canola, milho e soja por possuírem maior resistência térmica são indicados para processos que envolvam aquecimento como frituras, enquanto o azeite e o óleo de girassol são indicados para consumo ao natural como para o preparo de saladas.

\subsection{Palestra educativa nas escolas publicas e privada}

Foram desenvolvidas palestras sobre estabilidade oxidativa de óleos comestíveis e sobre doenças relacionadas a má alimentação como mostrada na Figura 1 para o público alvo que tiveram participação assídua com questionamentos e até mesmo com exemplos do seu cotidiano. 
FiguRA 1: PALESTRA SOBRE FORMAS DE UTILIZAÇÃo E DESCARTE DE ÓLEOS VEGETAIS.
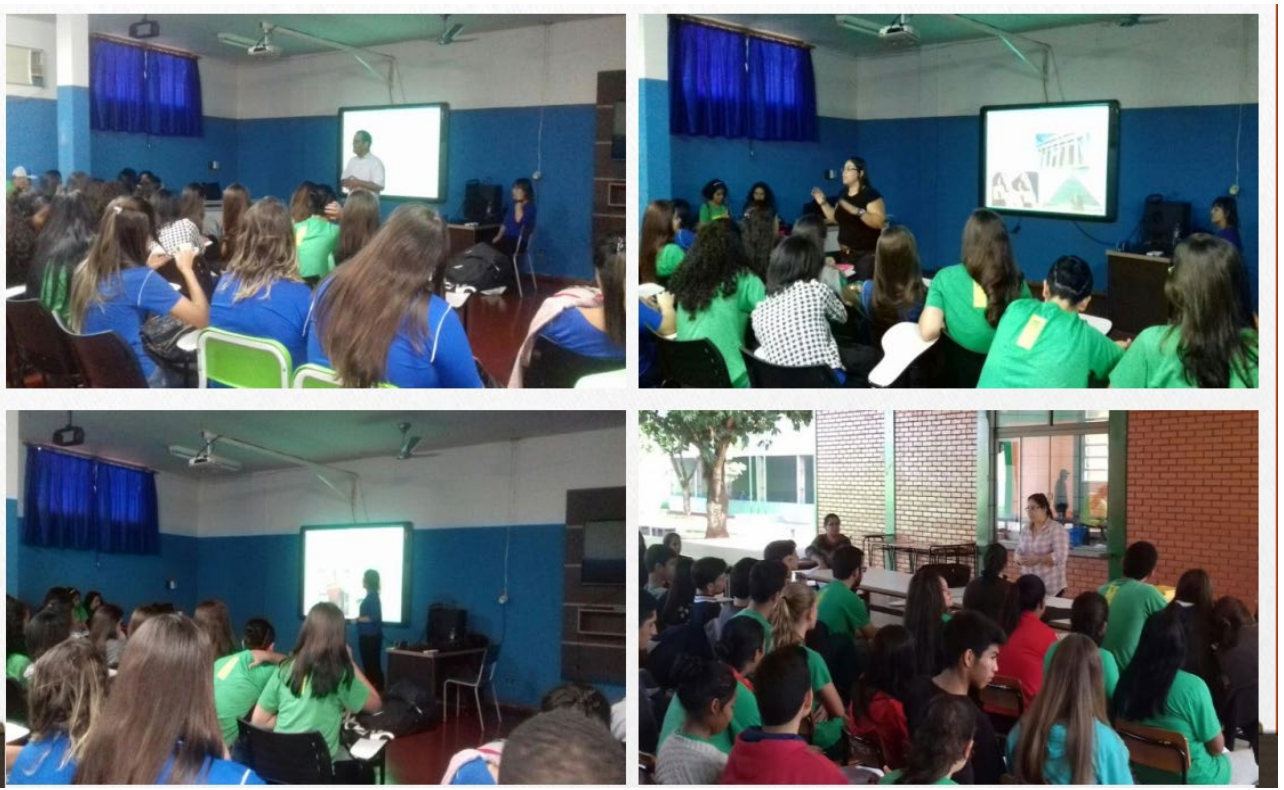

FONTE: AUTORES (2017)

As palestras foram preparadas para atender as principais necessidades dos grupos pesquisados sobre a reutilização dos óleos residuais, foram apresentadas ao público alvo técnicas de aproveitamento de resíduos oleosos para produção de diversos tipos de sabão, o desenvolvimento das atividades práticas pode ser observado nas Figura 2.

Figura 2: PRodução de SABÕes A PARTIR DE DESCARTE de Óleos VeGETAIS.
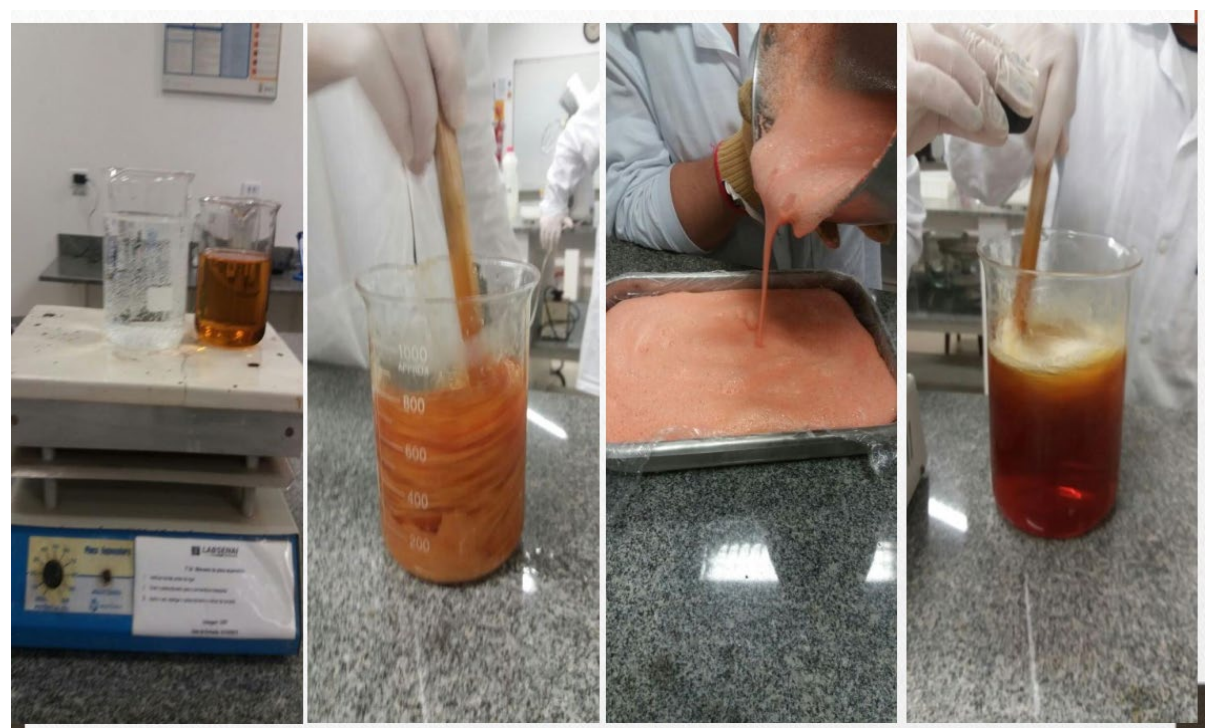

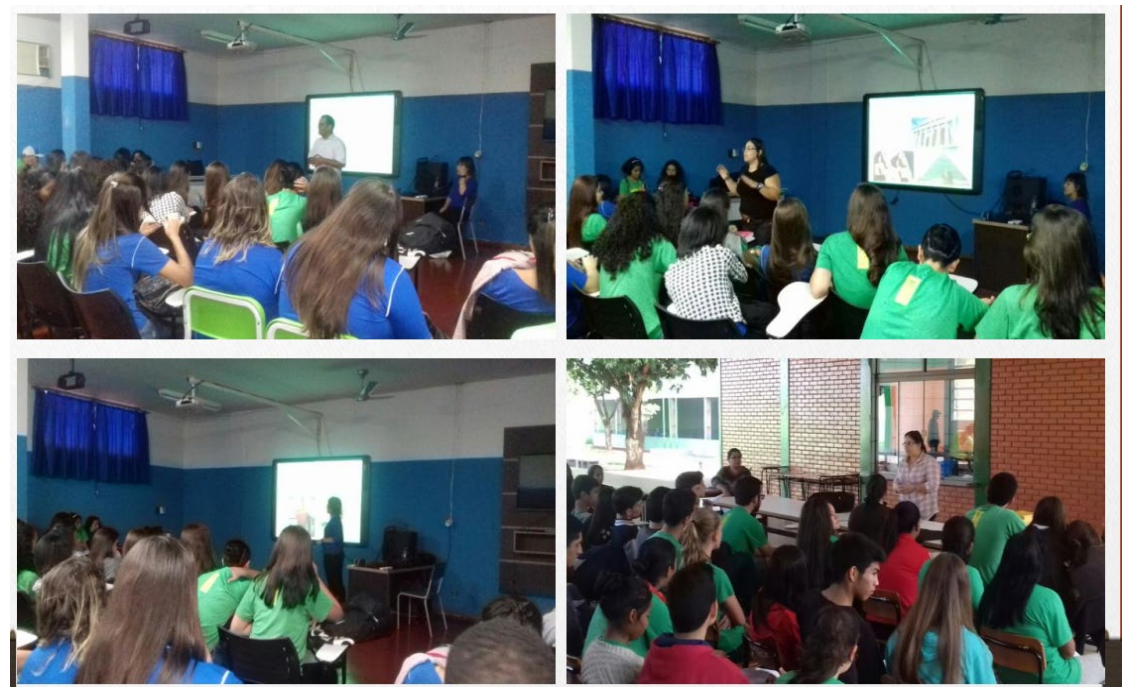

FONTE: AUTORES (2017)

Também foi oferecida uma visita técnica a Universidade. Os alunos do ensino técnico tiveram a oportunidade de conhecer diversos equipamentos utilizados para a pesquisa com óleos, aproximando a Universidade à comunidade conforme evidenciado na Figura 3.

Figura 3: Visita dos alunos do ensino técnico ao Centro de Estudos em Recursos Naturais(CERNA) da Universidade Estadual de Mato Grosso do SUL-DOURADOS MS.
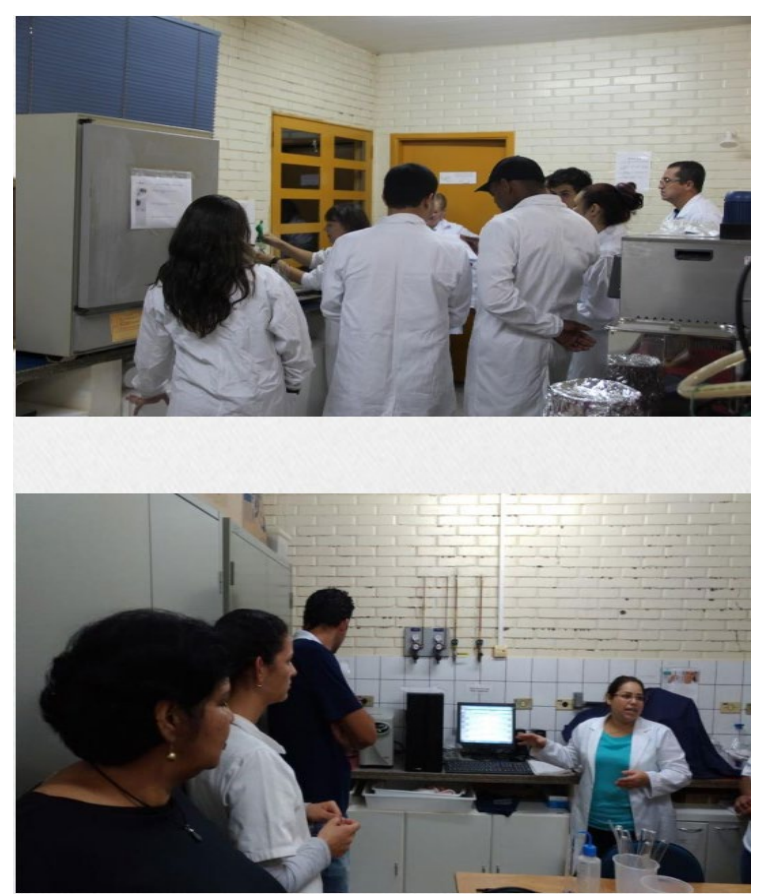

FONTE: AUTORES (2017). 


\subsection{Aplicação e análise do questionário}

O questionário foi disponibilizado para 300 entrevistados. O público alvo está dividido nos seguintes grupos:

- Alunos de ensino fundamental de escolas públicas de Dourados/MS.

- Alunos de ensino médio de escolas públicas de Dourados/MS

- Alunos de curso técnico do SENAI, Unidade Dourados/MS.

- Alunos de graduação da Universidade Estadual de Mato Grosso do Sul- Unidade Dourados/MS.

Os alunos com idade inferior a 18 anos levaram o questionário para que seus responsáveis respondessem. A primeira parte do questionário foi composta pela caracterização do perfil dos entrevistados. Foram perguntados o sexo, a idade, a renda e quantidade de pessoas que moravam com o entrevistado. A segunda parte visava a compreensão sobre a intensão de compra pelos possíveis consumidores de óleos comerciais, as perguntas eram sobre o tipo de óleo usado com maior frequência, o que determinava que o entrevistado optasse por um determinado tipo de óleo, questionava também sobre o conhecimento referente aos tipos de óleos existentes para consumo. Outro questionamento era referente a forma de utilização mais frequente do óleo no preparo de seus alimentos. Considerando o destino final após utilização dos óleos, foram questionados também sobre o que era feito com o óleo após a utilização, qual o critério utilizado pelo entrevistado para o descarte do óleo residual, indagava-se também sobre eventual reutilização do óleo e de que forma ela era feita.

O questionário com todas as perguntas é apresentado na Figura 4. 
FIGURA 4: QUESTIONÁRIO DE AVALIAÇÃO APRESENTADO AOS ALUNOS OU RESPONSÁVEIS.

\section{QUESTIONÁRIO DE AVALIAÇÃO}

Prezado(a). contamos com a sua gentileza para preencher o questionário abaixo, o que tomará apenas alguns minutos do sen tempo.

\section{$\underline{\text { 1. Perfil do consumidor }}$}

1.1 Sexo: ( ) M ( ) F

1.2 Idade:

( ) 15 a 20 anos

( ) 21 e 25 anos

( ) 26 e 30 anos

( ) 31 e 35 anos

( ) 36 e 40 anos

( ) 41 e 45 anos

( ) 46 e 50 anos

( ) acima de 50 anos

1.5 Qual o múmero de pessoas que moram na sua residência:

( ) 1 ( ) 2 a 3 ( ) 4 a 5 ( ) acima de 6

\section{Intencão de Compra}

2.1 Hoje você pretende comprar algum tipo de óleo comestivel?

( ) $\operatorname{sim}($ ) não ( ) não decidiu

2.2 Qual tipo de óleo comestivel adquire com maior frequência?

( ) soja ( ) girassol ( ) milho ( ) canola ( ) azeite de oliva ( ) outros

2.3 Qual fator que mais influencia na compra de um determinado tipo de óleo?

( ) preço ( ) de paladar (gosto, cheiro) ( ) aspectos nutricionais ( ) outros

2.4 Você tem algum conhecimento quanto a diferença de composição entre os diversos tipos de óleo?

( ) $\operatorname{sim}($ )não

2.5 Qual a forma de utilização mais frequente do óleo no preparo dos seus alimentos?

( ) fritura ( ) cozimento ( ) saladas ( ) refogados ( ) outros

2.6 Qual critério é utilizado na sua casa para decidir o momento de descarte do óleo de fritura?

( ) quantidade de vezes que foi utilizado ( ) tempo de utilização

( ) a spectos visuais e sensoriais

( ) outros

2.7 Na sua casa ocorre reutilizaçào para outras finalidades do óleo usado em frituras?

( ) sim ( ) nào

2.8 Se ocorre a reutilização, o que é feito com o óleo residual?

FONTE: AUTORES (2017).

3.3.1 Análise dos dados obtidos do questionário em relação ao perfil do consumidor

Os dados obtidos na primeira parte da análise do questionário aplicado no ensino fundamental podem ser observados a seguir na Figura 5, para o ensino médio na Figura 6, para o ensino técnico na Figura 7 e ensino superior na Figura 8. 
Figura 5: Ensino Fundamental, QUeStionário aplicado aos ResPonsáveis. PerFIL DO CONSUMIDOR.

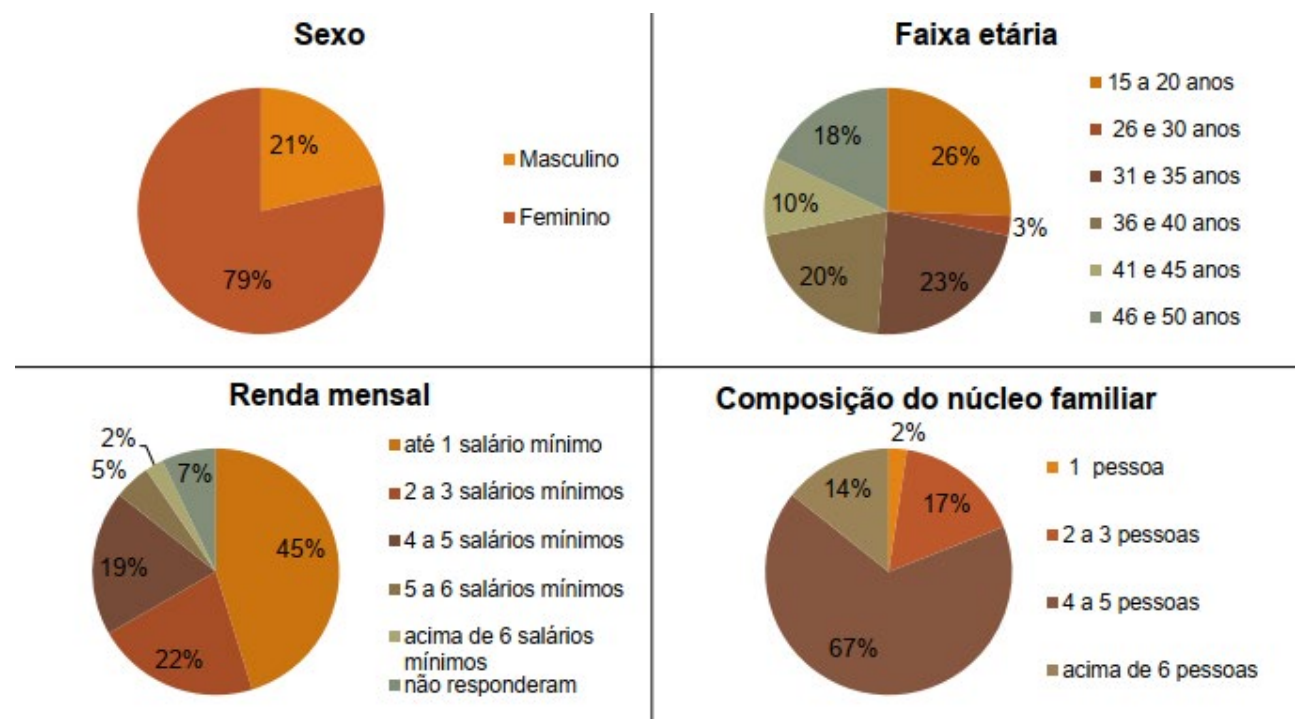

FonTE: AUTORES (2017).

Figura 6: Ensino médio, alunos/Responsáveis maiores de 18 anos. Perfil do CONSUMIDOR

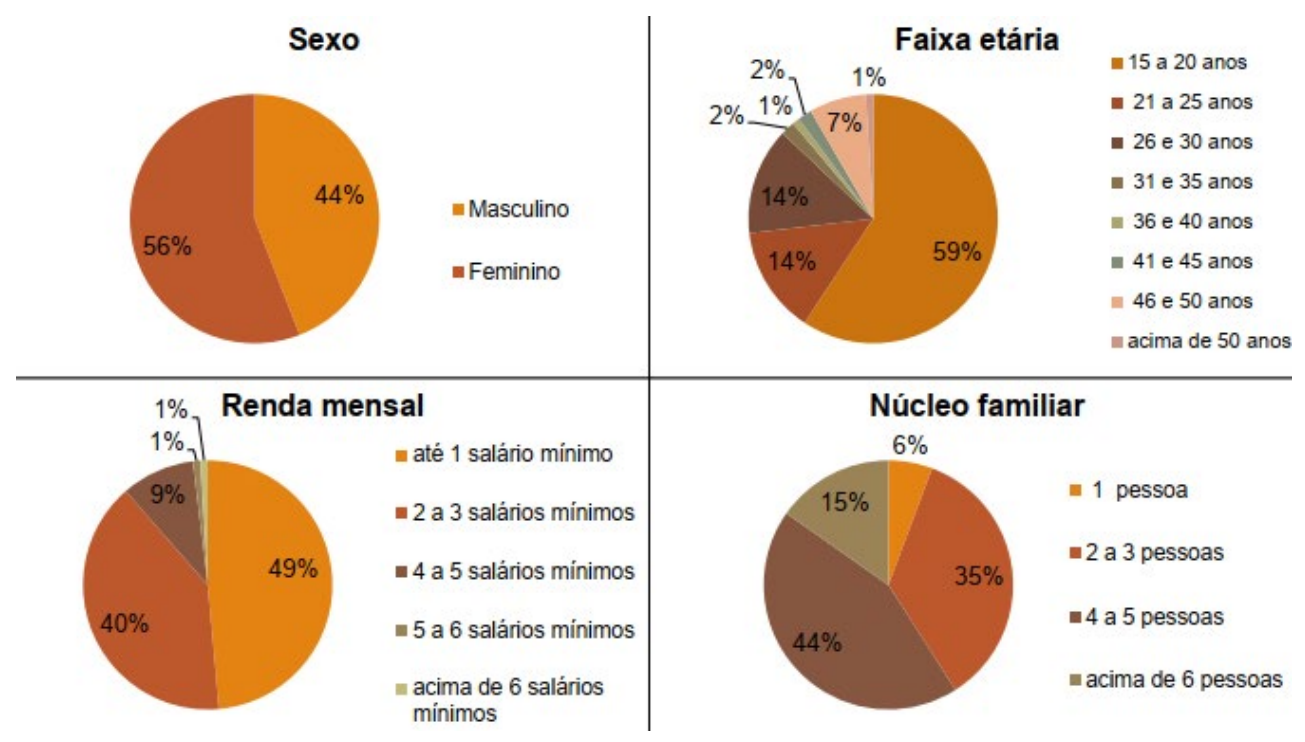

FONTE: AUTORES (2017) 
Figura 7: ENSINO TÉCNICO, ALUNOS MAIORES DE 18 ANOS. PERFIL DO CONSUMIDOR.

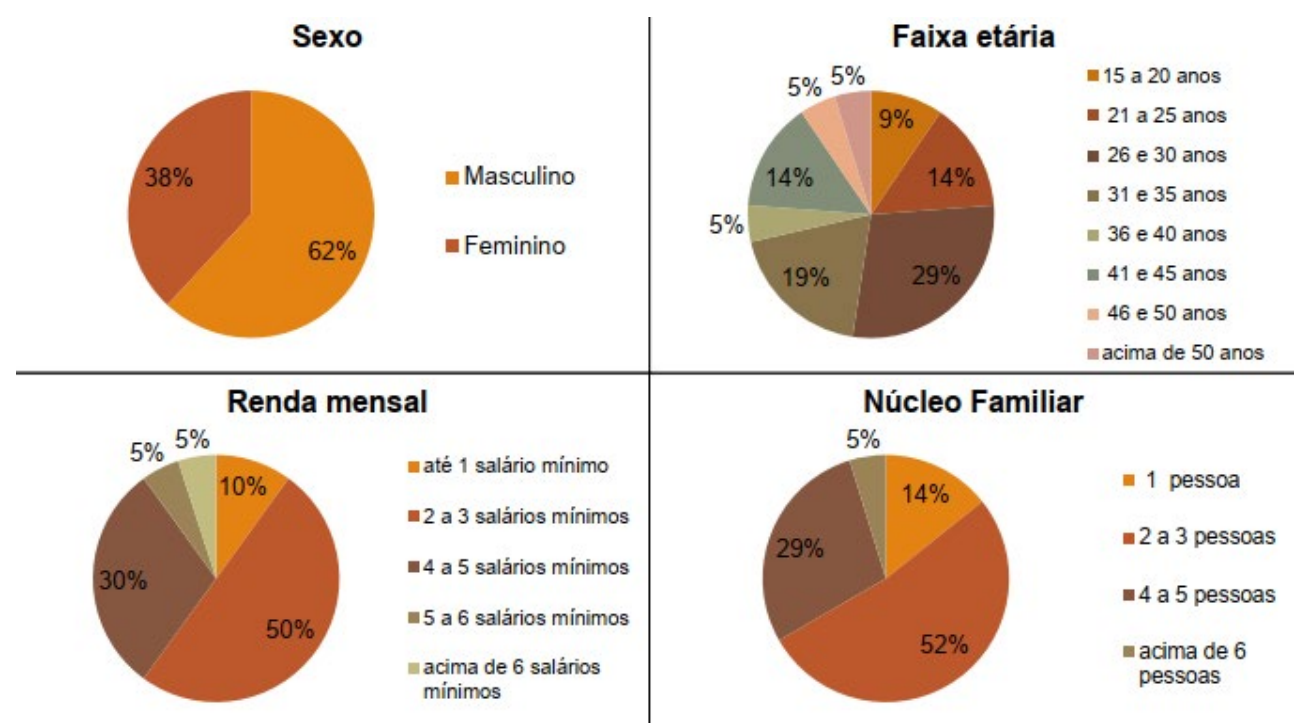

FONTE: AUTORES (2017).

Figura 8: ENSINO SUPERIOR, ACADÊMICOS DE DIFERENTES CURSOS. PERFIL DO CONSUMIDOR.

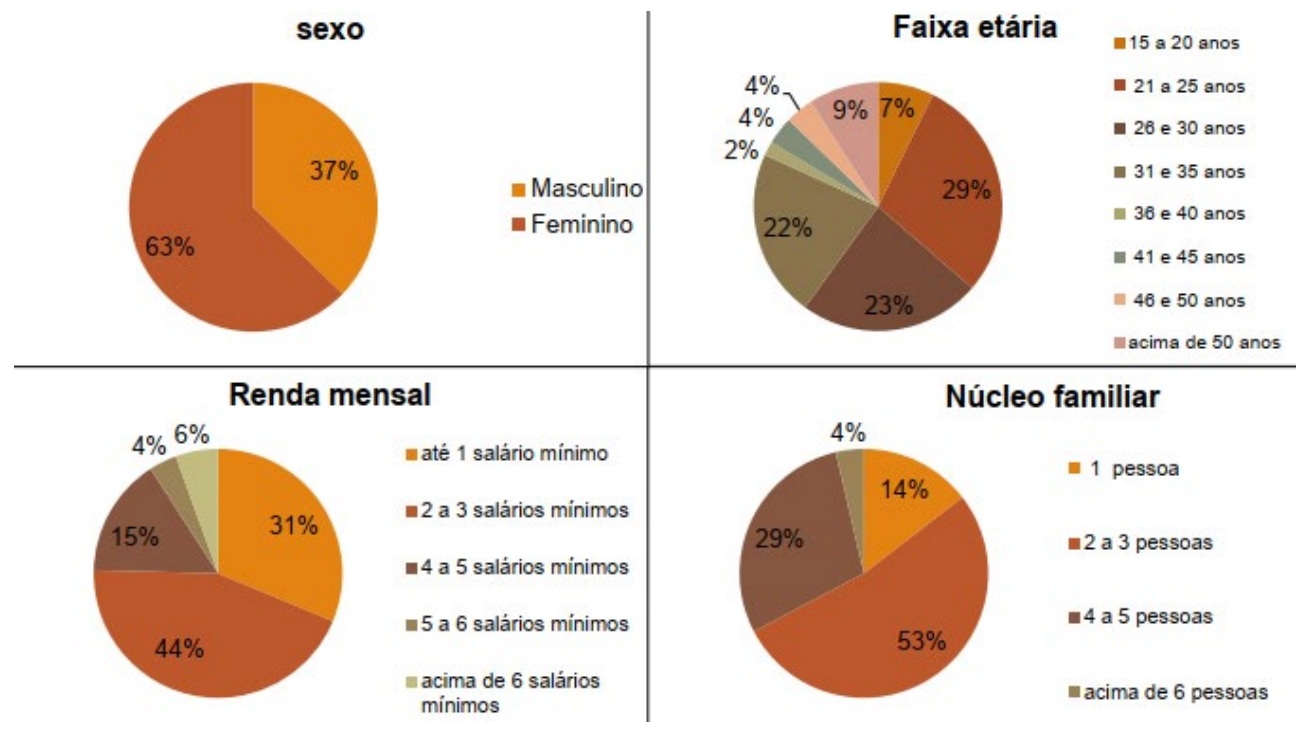

FONTE: AUTORES (2017).

Analisando os resultados do questionário mostrados nas Figuras 5 a 8 , percebe-se que apenas no ensino técnico a porcentagem de homens (62\%) respondendo o questionário foi superior ao de mulheres (38\%).

Quanto a pergunta sobre faixa etária no grupo de participantes do ensino fundamental, a maioria dos entrevistados são de idades entre 15 e 20 anos 
(26\%) seguido das idades de 31 a 35 anos (23\%), lembrando que alunos menores de 18 levaram o questionário para seus responsáveis responderem.

No grupo do ensino médio a maioria dos entrevistados apresentava idade entre 15 e 20 anos (59\%). Para os entrevistados no ensino técnico, as idades variavam entre 26 e 35 anos ( 29 e 19\%). Já no ensino superior a maioria com idades entre 21 e 30 anos (29\%).

A renda mensal dos entrevistados do ensino fundamental e médio predomina na faixa de até 01 salário mínimo (45 e 49\%), já os grupos de ensino técnico e superior estão na faixa de 2 a 3 salários mínimos (50 e 44\%).

O núcleo familiar dos entrevistados do ensino fundamental e médio é mais comum na faixa de 4 a 5 pessoas (67 e 44\%), já os grupos de ensino técnico e superior estão na faixa de 2 a 3 pessoas (52 e 53\%). Esses dados demostram as características de cada grupo entrevistado.

\subsubsection{Análise dos dados obtidos na segunda parte do questionário}

Os resultados obtidos com a análise da segunda parte do questionário aplicado no ensino fundamental podem ser observados na Figura 9, para o ensino médio na Figura 10, para o ensino técnico na Figura 11 e ensino superior na Figura 12.

Figura 9: ENSINO FUNDAMENTAL, QUESTIONÁRIO APLICAdO AOS RESPONSÁVEIS. INTENÇÃO DE COMPRA.

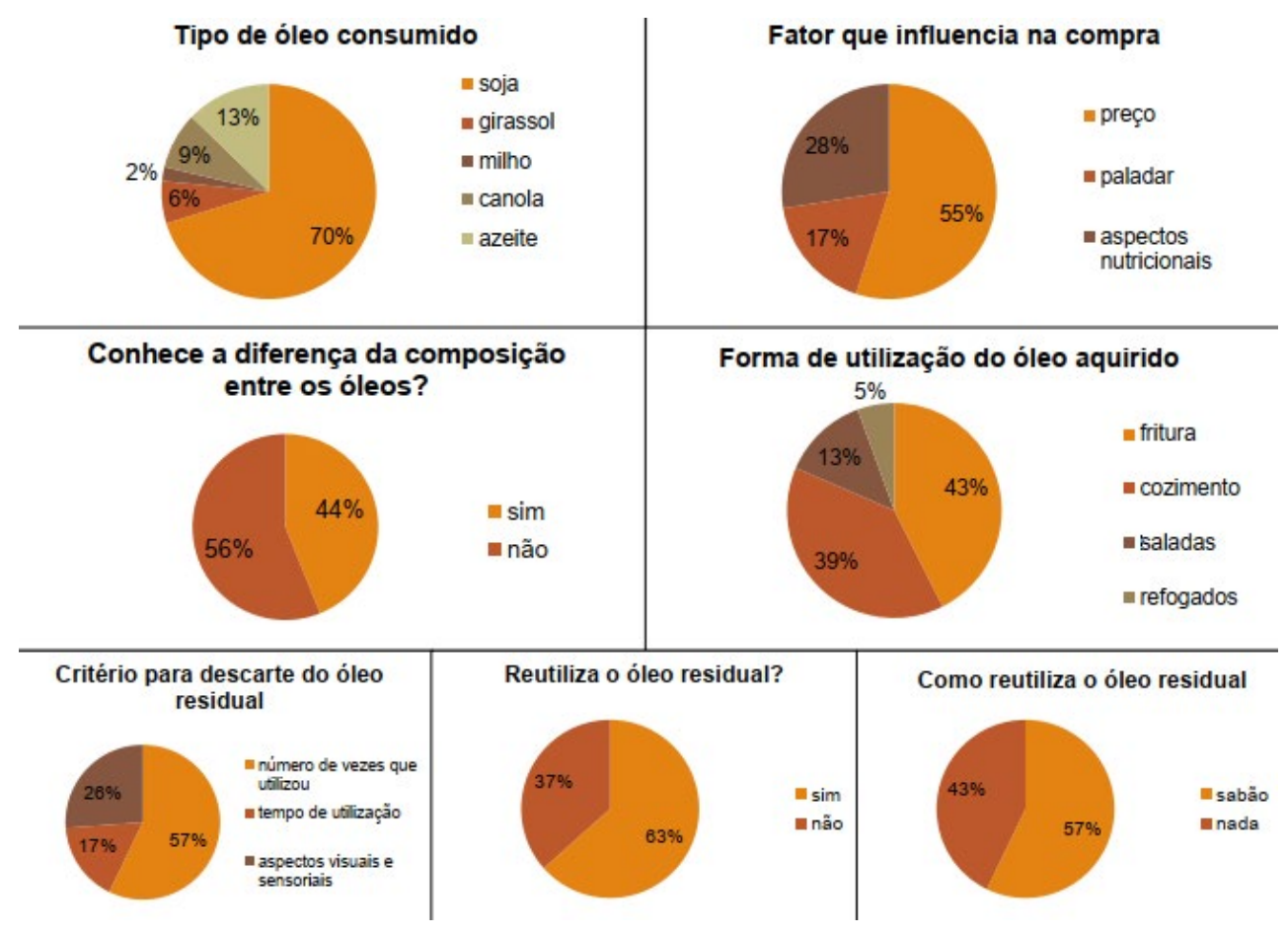

FONTE: AUTORES (2017) 
FIGURA 10: ENSINO MÉDIO, ALUNOS/RESPONSÁVEIS MAIORES DE 18 ANOS. INTENÇÃO DE COMPRAS

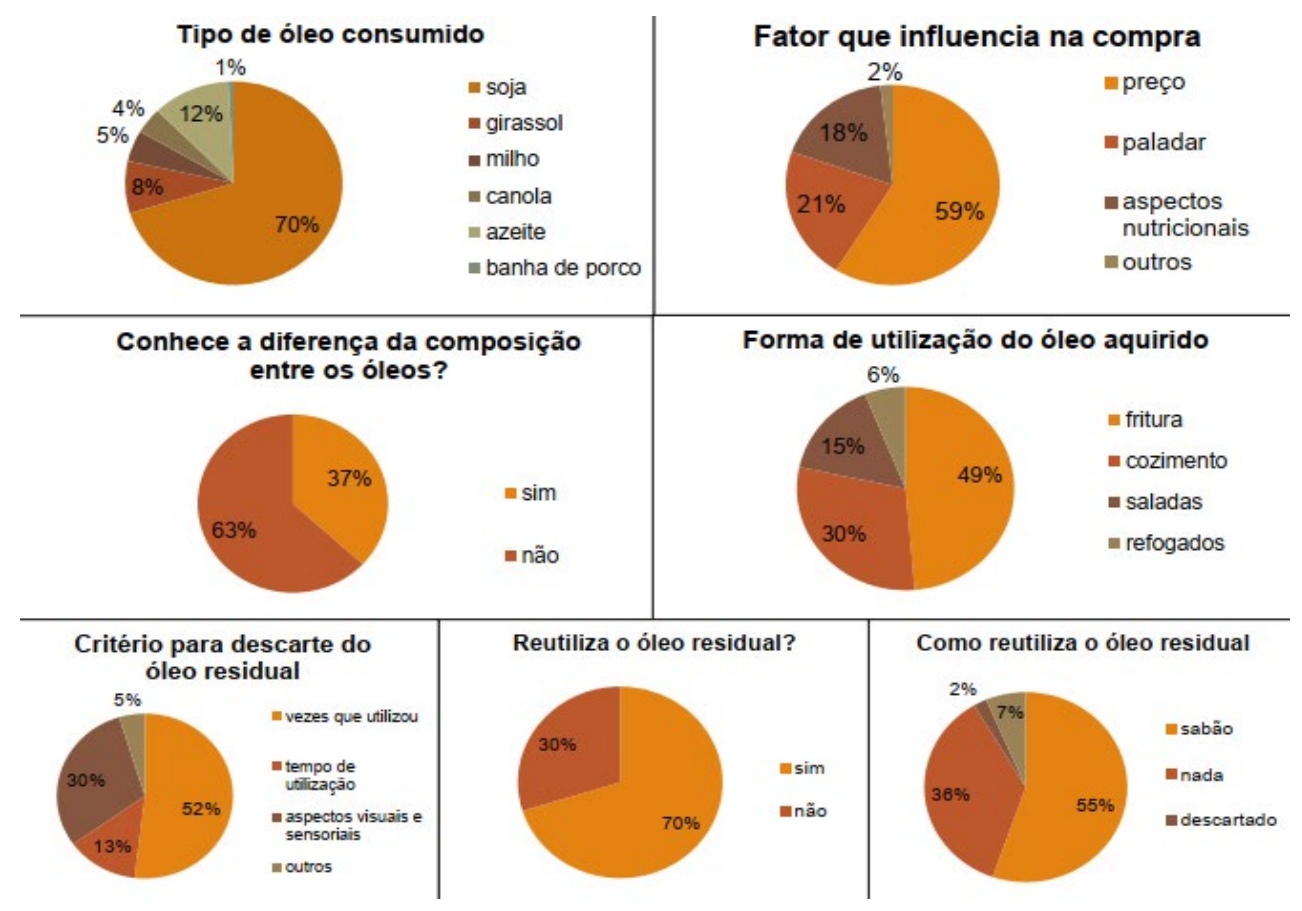

FoNTE: AUTORES (2017).

FIGURA 11: ENSINO TÉCNICO, ALUNOS/RESPONSÁVEIS MAIORES DE 18 ANOS. INTENÇÃO DE COMPRAS DE ÓLEO COMESTÍVEL.

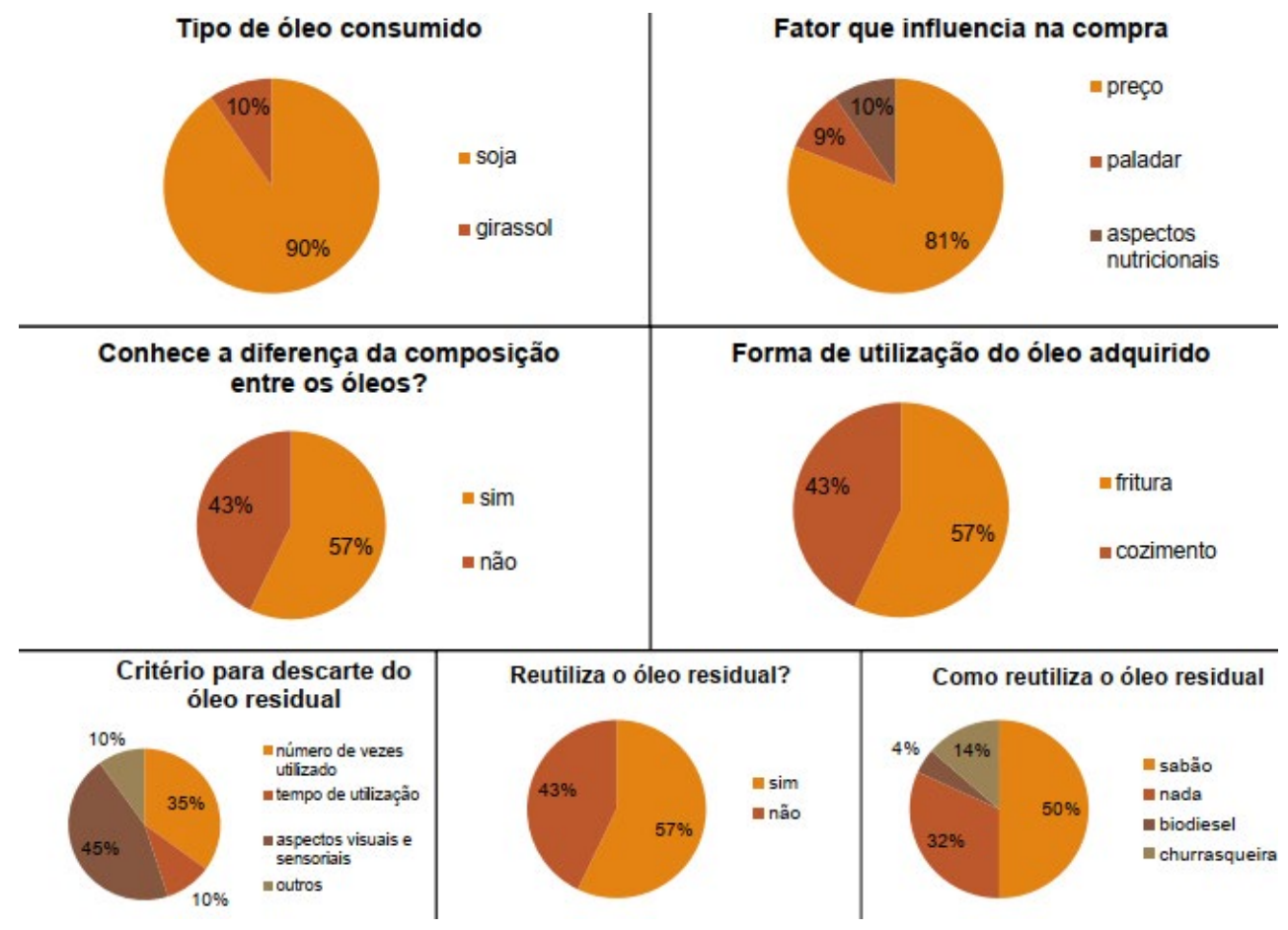

FonTE: AUTORES (2017). 
FIGURA 12: ENSINO SUPERIOR, ACADÊMICOS DE DIFERENTES CURSOS. INTENÇÃO DE COMPRA

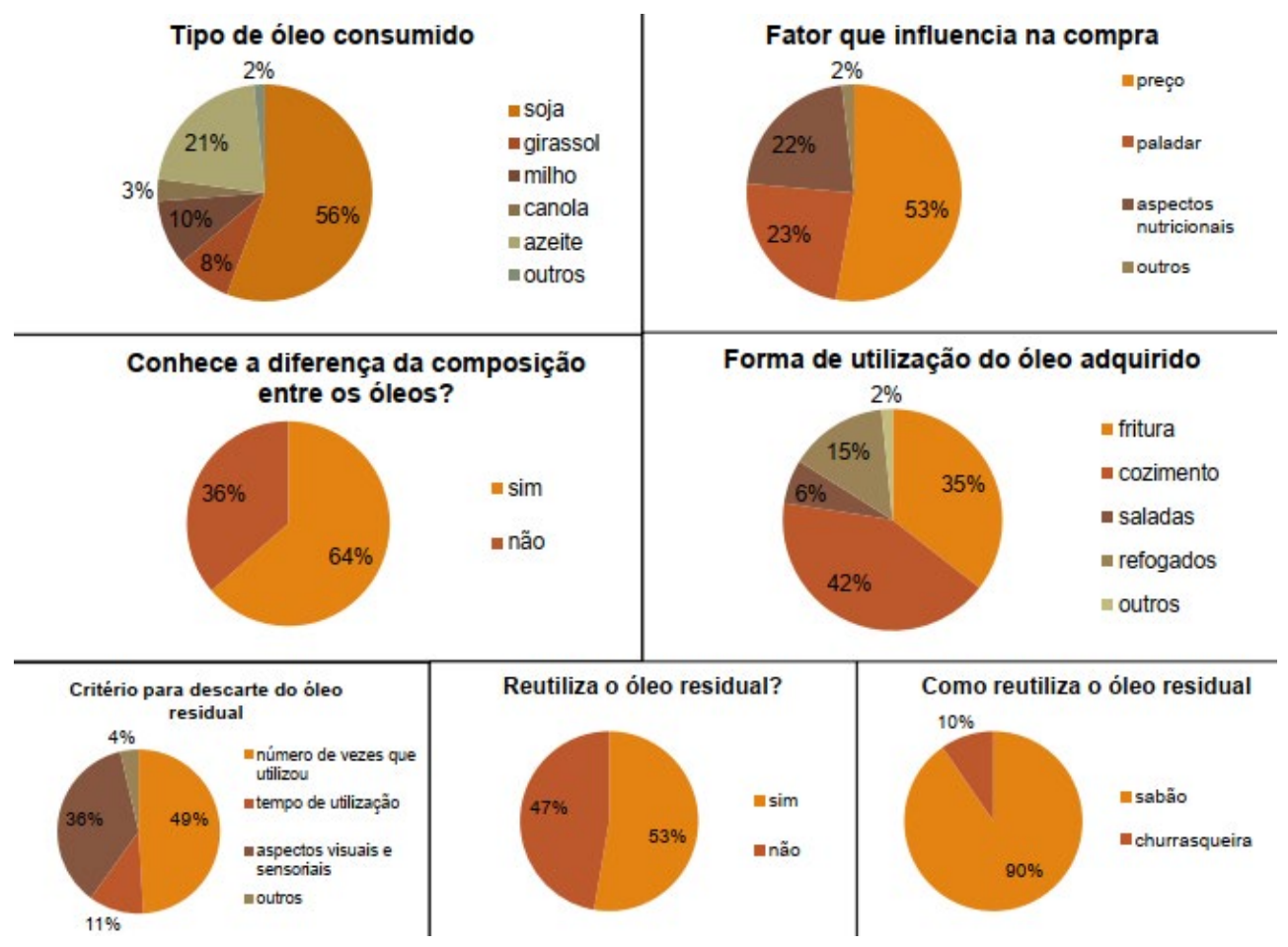

FONTE: AUTORES (2017).

Analisando os resultados do questionário das Figuras 9 a 12, observa-se que independente do perfil do consumidor, em relação a idade ou renda, a maioria das pessoas entrevistadas fazem uso do óleo de soja (70, 70, 90 e 56\%) e levam em consideração o valor econômico $(55,59,81$ e 53\%) para fazer essa escolha.

Segundo Castro (p. 81, 2016) "Apesar de ocupar o segundo lugar na produção total de óleos vegetais, o óleo de soja é o primeiro em consumo na alimentação humana, resultado da expressiva produção da oleaginosa no mundo." Informação que vai ao encontro do maior percentual de consumo pelos entrevistados neste estudo.

Constatou-se também nos questionários respondidos pelos responsáveis dos alunos do ensino fundamental e pelos alunos do ensino médio que menos de $50 \%$ dos entrevistados não tem conhecimento da diferença na composição dos óleos comerciais. Já no ensino técnico e superior ocorre o contrário, a maioria das pessoas (57 a 64\%) que responderam ao questionário tem conhecimento sobre a diferença de composição. Isso confirma a impotência das palestras realizadas sobre essa temática nas escolas participantes do 
desenvolvimento da atividade realizada influenciando mudanças de comportamento dos alunos e familiares.

O projeto de extensão universitária aqui desenvolvido possibilitou a comunidade novos conhecimentos científicos e tecnológicos que podem se refletir em_melhoria na qualidade de vida.

\begin{abstract}
A Extensão Universitária possui papel importante no que se diz respeito às contribuições que pode trazer frente à sociedade. É preciso, por parte da Universidade, apresentar concepção do que a extensão tem em relação a comunidade em geral. Colocar em prática aquilo que foi aprendido em sala de aula e desenvolvê-lo fora dela. A partir do momento em que há esse contato entre o aprendiz e a sociedade beneficiada por ele, acontece por parte dos dois lados, benefícios. Aquele que está na condição do aprender acaba aprendendo muito mais quando há esse contato, pois torna-se muito mais gratificante praticar a teoria recebida dentro da sala de aula. Esse é o conceito básico de extensão. (RODRIGUES et.al. p. 142, 2013)
\end{abstract}

Durante as palestras, os alunos puderam esclarecer dúvidas referente a composição dos óleos, diferenças sobre a estabilidade oxidativa dos óleos pesquisados, conhecimento esse que poderá ser compartilhado com seus familiares e responsáveis.

Outra análise importante é sobre a reutilização dos óleos, a maioria dos entrevistados de todos os grupos analisados alegaram reutilizar o óleo residual (53 a 70\%) e a maioria leva em consideração a quantidade de vezes que o óleo foi usado (35 a 57\%). Essa prática deve ser feita com cuidado, pois estudos demonstram o perigo da reutilização. O óleo reutilizado, quando aquecido excessivamente apresenta efeito negativo sobre o organismo humano devido a ocorrência da formação do composto de acroleína e a decomposição da gordura.

Silva et al. (2017) em seu estudo analisaram a fumaça de óleo de canola, girassol, dendê e azeite extra virgem de oliva e concluíram que o composto acroleína derivado do aquecimento prolongado de óleos e gorduras aumenta expressivamente quando a temperatura de preparo dos alimentos é superior à $180^{\circ} \mathrm{C}$. Segundo os mesmos autores esses resultados explicam a relação do consumo de alimentos fritos com o surgimento de doenças como o câncer, principalmente por estarem relacionados a ingestão involuntária de acroleína.

O descarte do óleo residual também é um fator de estudo relevante observou-se que os entrevistados possuem duas principais práticas para o descarte dos óleos que são elas, a fabricação de sabão (50 a 90\%) ou simplesmente não fazem nada com o óleo residual (32 a 42\%). 
Segundo a Legislação Ambiental, o tema "óleo de cozinha” está sendo abordado pelo projeto de Lei no 2.074 de 19 de setembro de 2007 em tramitação no Congresso Federal Brasileiro, em diversas discussões para tratar do destino deste resíduo que vem causando grandes danos ambientais, quando descartado de forma inadequada pelos lares, microempresários do ramo de salgadinhos fritos, lanchonetes e restaurantes, entre outros. (COSTA, LOPES e LOPES, p. 246, 2015)

Constatou-se com as respostas do questionário que uma grande parte dos entrevistados utiliza o óleo residual para fabricação de sabão; esta é uma solução para o descarte inadequado evitando danos ao meio ambiente.

O sabão fabricado com óleo residual de acordo com Nascimento et al. (2010), possui taxa de degradação maior que os sabões vendidos comercialmente, além de não apresentar em sua composição o elemento químico fósforo que é um dos compostos de maior índice de poluição e propagação de algas tóxicas, modificando também cor, odor e sabor da água.

\section{CONCLUSÕES}

O projeto atingiu o público alvo induzindo possíveis mudanças no setor social, educacional e ambiental. Por meio dos resultados das análises laboratoriais os alunos evidenciaram que os óleos de canola, milho e soja possuem maior resistência térmica sendo indicados para processos que envolvam aquecimento como frituras, enquanto o azeite e o óleo de girassol são indicados para consumo ao natural como o preparo de saladas. Foi apresentada também aos alunos do ensino fundamental e médio uma nova perspectiva de utilização do óleo residual. Esses alunos possivelmente serão influenciadores de mudanças de atitude no núcleo familiar e social.

Quanto aos alunos do ensino técnico a proposta de utilização de óleo residual pode gerar ainda uma possibilidade de incremento financeiro, pois os mesmos estão aptos a desenvolver novos produtos. Além disso, para esse grupo houve uma aproximação com a Universidade pública, incentivando os mesmos a procura de novas oportunidades na Universidade e oportunizando conhecimento de uma técnica de análise de óleos para avaliação de sua estabilidade oxidativa. 


\section{AGRADECIMENTOS}

A Fundação de Apoio ao Desenvolvimento do Ensino, Ciência e Tecnologia do Estado de Mato Grosso do Sul (FUNDECT), ao Conselho Nacional de Desenvolvimento Científico e Tecnológico (CNPq), ao Programa Institucional de Bolsas aos Alunos de Pós-Graduação (PIBAP-UEMS), a Coordenação de Aperfeiçoamento de Pessoal de Nível Superior (CAPES) pelo financiamento dos equipamentos (Edital CAPES No 11/2009 - Pró-Equipamentos Institucional) e as escolas participantes do projeto (rede pública/privada).

\section{REFERÊNGIAS BIBLIOGRÁFICAS}

ANVISA-Agência Nacional da Vigilância Sanitária. RDC N 593, de 25/08/2000. Disponível em: www.anvisa.gov.br. Acesso em: 08/04/2017.

ARAÚJO, A. M. de M. Conversão térmica e termocatalítica à baixa temperatura do óleo de girassol para obtenção de bioóleo. Dissertação (Mestrado) - Universidade Federal do Rio Grande do Norte, 2012. Centro de Ciências Exatas e da Terra. Programa de Pós-Graduação em Ciência e Engenharia de Petróleo. Disponível em: <http://www.nupeg. ufrn.br/documentos_finais/dissertacoes_de_mestrado/dissertacoes/aruzzamabel. pdf> Acesso em: 18 abr. 2019.

BRASIL. Ministério da Saúde. Secretaria de Atenção à Saúde. Departamento de Atenção Básica. Política Nacional de Alimentação e Nutrição / Ministério da Saúde, Secretaria de Atenção à Saúde. Departamento de Atenção Básica.Básica. - 1. ed., 1. reimpr. Brasília: Ministério da Saúde, 2013.

BODEIRA, R. M.; PENCI, M. C.; RIBOTTA, P. D.; MARTÍNEZ, M. L. Chia (Salvia hispanica L.) oil stability: Study of the effect of natural antioxidants. LWT - Food Science and Technology, 75, 107, 2017. http://dx.doi.org/10.1016/j.lwt.2016.08.031

CASTRO, L. S. de. Analisando a substitutibilidade no mercado mundial de óleos vegetais via transmissão de preços. CONTEXTUS Revista Contemporânea de Economia e Gestão, Fortaleza-CE, 2016. Disponível em: <http://periodicos.ufc.br/contextus/article/ view/32276/pdf.> Acesso em: 2 maio 2019.

CORSINI M. da S., JORGE, N. Estabilidade oxidativa de óleos vegetais utilizados em frituras de mandioca palito congelada. Ciênc. Tecnol. Aliment, Campinas, 26(1): 27-32, jan.-mar. 2006.

COSTA, D. A. da; LOPES, G. R.; LOPES, J. R. Reutilização do óleo de fritura como uma alternativa de amenizar a poluição do solo. Revista Monografias Ambientais - REMOA, Santa Maria, 2015. Disponível em: <https://periodicos.ufsm.br/remoa/article/ view/20461/pdf > Acesso em: 26 abr. 2019. 
FARHOOSH, R.; EINAFSHAR, S.; SHARAYEI, P. The effect of commercial refining steps on the rancidity measures of soybean and canola oils. Food Chemistry, 115, 933-938, 2009.

FARHOOSH, R.; MOOSAVI, S. M. R. Rancimat test for the assessment of used frying oils quality. Journal of food lipids, 14, 263-271, 2007.

FIRESTONE, D.; STIER, R. F.; BLUMENTHAL, M. M. Regulation of frying fats and oils. Food Technology, 45, 90-94, 1991.

FOOD INGREDIENTS BRASIL. OS LIPÍDIOS E SUAS PRINCIPAIS FUNÇÕES. Revista-FI, No 37 - 2016. Disponível em: <http://www.revista-fi.com/materias/541.pdf> Acesso em: 08/04/2017.

FUENTES, P, H. A. Avaliação da qualidade de óleos de soja, canola, milho e girassol durante o armazenamento. 2011. Dissertação (Programa de Pós-Graduação em Ciência dos Alimentos) - Aluno, FLORIANÓPOLIS, 2011. Disponível em: $<$ https://repositorio. ufsc.br/bitstream/handle/123456789/95494/297094.pdf>. Acesso em: 18 abr. 2019.

JUN. A. Reatividade de Compostos Orgânicos II e Biomoléculas. Lipídios, ácidos graxos e fosfolipídeos. USP -Instituto de Química. Disponível em: <https://edisciplinas.usp. br/pluginfile.php/2274192/mod_resource/content/0/Resumo_08_Gr10.pdf $>$ Acesso em: 08/04/2019.

LEONARDO, R da S. Estudo da estabilidade de biodiesel com misturas de antioxidantes por um método de p-dsc de baixa pressão. 142 f. Dissertação (Mestrado) - Curso de Mestre em Ciências, Universidade Federal do Rio de Janeiro, Rio de Janeiro, 2012. Cap. 2 MELO, W. V. de; BIANCHI, C. dos S. Discutindo estratégias para a construção de questionários como ferramenta de pesquisa. R. B. E. C. T, São Gotardo, 2015. Disponível em: <https://periodicos.utfpr.edu.br/rbect/article/view/1946/2179> Acesso em: 2 maio 2019.

MENDONÇA, M. A.; BORGO, L. A.; ARAÚJO, W. M. C.; NOVAES, M. R. C. G. Alterações físico-químicas em óleos de soja submetidos ao processo de fritura em unidades de produção de refeição no Distrito Federal. Com. Ciências Saúde, 19, 2, 115-122, 2008.

NASCIMENTO, Antônio Carlos M. et al. A logística reversa do óleo de fritura usado como solução para problemas ambientais. 2010. Disponível em: $<$ http://www.setorreciclagem.com.br/images/oleo.pdf> Acesso em: 3 maio 2019.

OSAWA, C. C.; Gonçalves, L. A. G.; Mendes, F. M. (2010). Avaliação dos Óleos e Gorduras de Fritura de Estabelecimentos Comerciais da Cidade de Campinas/SP. As Boas Práticas de Fritura Estão Sendo Atendidas? Alimentos e Nutrição, v. 21, p. 47- 55, 2010.

REDA, Y. S.; CARNEIRO, P. I. B. Óleos e gorduras: Aplicações e implicações. Revista Analytica, 27, 60-67, 2007

RODRIGUES, A. L. L. et al. Contribuições da extensão universitária na sociedade. Cadernos de Graduação - Ciências Humanas e Sociais , ARACAJU, 2013. Disponível em: https://periodicos.set.edu.br/index.php/cadernohumanas/article/viewFile/494/254. Acesso em: 2 maio 2019. 
MARTINS, J. S.; FREIRE, M. S.; DA SILVA NETO, N.; SILVA, T. N.; SANTOS, J. C. O. Caracterização físicoquímica do óleo de coco obtido artesanalmente. $5^{\circ}$ Congresso Norte-Nordeste de Química - UFRN, Rio Grande do Norte, 2013.

SANTOS, V. S. Desenvolvimento de barras de alto teor proteico a partir da castanha do Brasil. Dissertação de Mestrado, Ciência e Tecnologia de Alimentos - Universidade Federal do Pará, Belém, p. 95, 2008.

SILVA, A. F. da et al. Relação entre acroleína e câncer: uma revisão. In: V CONGRESSO DE PESQUISA E EXTENSÃO DA FSG III SALÃO DE EXTENSÃO, 2017, Caxias do Sul - RS. 2017. Disponível em: <http://ojs.fsg.br/index.php/pesquisaextensao/article/ view/2652> Acesso em: 26 abr. 2019.

SILVA NETO N, SANTOS, J. R. M.; MARTINS, J. S.; FREIRE, M. S.; SANTOS, J. C. O. Caracterização química e físicoquímica do óleo de coco extra virgem (Cocos nucifera L.). $5^{\circ}$ Congresso Norte-Nordesde de Química. $3^{\circ}$ Encontro Norte-Nordeste de Ensino de Química, Universidade Federal do Rio Grande do Norte, 2013.

SUAREZ, P. A. Z. MENEGHETTI, S. M. P.; MENEGHETTI, M. R.; WOLF, C. R. Transformação de triglicerídeos em combustíveis, materiais poliméricos e insumos químicos: algumas aplicações da catálise na oleoquímica. Quim. Nova, Vol. 30, No. 3, 667-676, 2007. TAN, C. P.; CHE MAN, Y. B.; SELAMAT, J.; YUSOFF, M. S. A. Comparative studies of oxidative stability of edible oils by differential scanning calorimetry and oxidative stability index method. Food chemistry, 76, 385-389, 2002. 